\title{
Monika Wolting* \\ FABIAN WOLBRING: DIE POETIK DES DEUTSCHSPRACHIGEN RAP. V\&R UNIPRESS, GÖTTINGEN 2015, SS. 627
}

Würde man die begriffliche Verständigung über Popliteratur mit einer ebenso möglichen Diskussion über Popmusik vergleichen, dann würde man zu erstaunlichen Ergebnissen kommen: Die fiktionalen Texte des Rap wurden noch keiner präzisen Klassifizierung unterzogen. Während also Popliteratur als Oberbegriff eine zwar vage, aber letztlich doch klar umrissene Gattung beschreibt - sie reicht von der Beatliteratur über Rolf Dieter Brinkmann zu Rainald Goetz und Thomas Meinecke und von Nick Hornby über Benjamin von Stuckrad-Barre und Christian Kracht bis hin etwa zu Judith Hermann, Thomas Brussig oder Sibylle Berg -, war bis jetzt eine ähnliche Verwendung der Subgattung der Rap-Texte kaum denkbar. Gerade in dieser Gattung, in dem Rap, geben sich die POP-Literatur und die POP-Musik die Hand. Es wird an der Zeit, dass dies laut gesagt wird und durch eine fundierte literaturwissenschaftliche Untersuchung bestätigt wird, dass RAP eine besondere Stellung in der deutschsprachigen Gegenwartsliteratur annimmt, ihm soll der Platz der einflussreichen Lyrikform der Gegenwart überhaupt zugeschrieben werden.

Fabian Wolbring verfasst einen 627 Seiten starken Band mit dem Titel „Die Poetik des deutschsprachigen Rap“(V\&R unipress 2015). Der Autor ist nicht nur Literatur- und Rapwissenschafter, aber auch ein langjähriger Rapschaffender.

Der Band liefert eine der ersten literaturwissenschaftlichen Abhandlungen zu dem Thema. Auch aus diesem Grund ist es von Bedeutung etwas genauer in den Band zu schauen, denn er könnte eine Vorlage für weitergehende literatur- und kulturwissenschaftliche Beschäftigung mit dem Thema aufbereiten.

Durch die Behandlung des Rap als Lyrikform und die Fokussierung auf die poetische Praxis seiner Produktion schafft Wolbring einen neuen Zugang zur Erforschung der Gattung. Der Autor definiert überzeugend den Rap als Lyrikform. Wolbring behandelt die Rap-Texte als Gedichte und damit als ästhetische Texte. Solche Herangehensweise ist in der Forschung bislang nur rudimentär vorhanden.

* Dr hab. Monika Wolting, Instytut Filologii Germańskiej, Uniwersytet Wrocławski, Pl. Biskupa Nankiera 15, 50-140 Wrocław. e-mail: monikawolting@yahoo.de 
Versteht man $P O P$ als Kurzwort mit der Bedeutung populär, gibt der Autor zu bedenken, dann ist Rap eine Form des Pop. Er ist auch der Überzeugung, dass gerade weil Rap einen popkulturellen Einfluss ausübt, sollte er als Gegenstand der Pop-Forschung zu klassifizieren sein.

Wolbring behandelt in seinem Band Raps als Sprechgedichte. Seine Behauptung setzt er an der Überlegung fest, dass er zum einen die Prädominaz der Sprache im Rap-Song feststellt, zum anderen dass inzwischen das Selbstverständnis der Rapschaffenden als Sprachkünstler in den einschlägigen literaturwissenschaftlichen Beiträgen bestätigt wird.

In dem eigentlichen Hauptteil der Arbeit widmet sich der Autor zwei unterschiedlichen Zugängen zu der Gattung. Zunächst wird das Rap-Schaffen als poiesis und damit als literarische Praxis bestimmt. Hierzu erklärt Wolbring das gängige Autorschaftsmodell, nach dem sowohl die nach regelpoetischen Standards messbare Kunstfertigkeit, als auch die Erfüllung impliziter Authentizitätsversprechen die Qualität eines Autors ausmachen. Bei der anschließenden Beschreibung des Rapschaffenden als sprachliche Mischtätigkeit mit oralen und literalen Anteilen orientiert sich der Verfasser an medientheoretischen Überlegungen.

Im zweiten Teil stellt er die signifikanten Kennzeichen der Textsorte Rap vor. Dabei werden mediale, formale und inhaltliche Aspekte, sowie ihr Wirkungszusammenspiel berücksichtigt. Wolbring betont die besondere Leistung der Rap-Künstler in der Wortschatzarbeit. Die Rappschaffenden sind ständig bemüht, neue und unverbrauchte Formulierungsalternativen zu sammeln und zu generieren. Neben der unmittelbaren Neologismusbildung werden vor allem auch abseitige, ungewöhnliche und dennoch allgemeinverständliche Neologismen in den Text integriert. Eins der wichtigsten Kennzeichnen des Rap sind nach Wolbring die Flow-Modulationsmöglichkeiten und Sprechrhythmen. Um dieses komplizierte Thema auszuloten, liefert Wolbring mehrere Beispiele, an denen er die variable Sprechgeschwindigkeit, Akzentsetzung wie rhythmische Muster aufzeigt und analysiert. Wolbring stellt weiter heraus, dass im ambitionierten Rapschaffen sogenannte Standardreime allgemein abgelehnt werden. Die Künstler bemühen sich stattdessen um exotische bzw. seltene Reime, nach Möglichkeit sogar neuartige Reime. So stellt der Autor fest, dass die beiden wesentlichen kennzeichnenden Stilmittel des Rap der Sprachrhythmus und der Reim sind. Er bemerkt jedoch dazu, dass diese in der klassischen Rhetorik nicht dezidiert verhandelt werden. Die Ursachen für diesen Zustand findet er in ihrer primär formalen bzw. ästhetischen Funktion.

Die Textsorte Rap ist im Verständnis des Autors durch formal-ästhetische Kennzeichen bestimmt, ohne die sich Raps gar nicht als solche erkennen ließen. Er ist der Auffassung, dass eine inhaltliche oder ideologische Ausrichtung durch die Textsorte nicht zwingend vorgegeben wird. Raps werden zu allen möglichen Themen verfasst, nach dem Motto: „Raps passen zu jeder Lebenssituation“. Die thematische Offenheit wird dann aber als eine besondere Qualität dieser Texts- 
orte ausgestellt. Der Autor konnte nachweislich machen, dass die Textsorte besonders dazu geeignet ist, ihre Sprecher als cool und überzeugend ins Szene zu setzten.

Der letzte Teil der Abhandlung, unter dem Titel „Abschließende Bemerkungen zum gesellschaftlichen Umgang mit Rap“ gewinnt einen kulturwissenschaftlichen Mehrwert. Denn hier resümiert der Autor über die gesellschaftliche Bedeutung des Rap in Deutschland. Dabei kommentiert er potenzielle Motive für das Rapschaffen, Probleme und Gefahren, die sich aus ihm ergeben und schildert seine gesellschaftliche Aufgabe. Wolbring konstatiert: „Meines Erachtens fehlt es vor allem an einem ästhetischen Diskurs, der das Rapschaffen als literarische Praxis ernst nimmt, ohne es dabei zu glorifizieren. (...) Das Rapschaffen ist eine bislang weitestgehend ungenutzte Möglichkeit, einen breiten Lyrik-Diskurs anzustoßen.“ (499)

Wolbring unterzieht einer literaturwissenschaftlichen Analyse einen enormen Textkorpu. Diese Arbeit gilt zum einen als Basis für die Erstellung der Typologie der Texte, zum anderen aber auch archiviert der Autor Unmengen von Textbeispielen für eine weitere mögliche Analyse. Diese Abhandlung sollte hauptsächlich als eine Pionierarbeit auf dem Feld der literaturwissenschaftlichen Forschung zu Rap-Texten gelesen werden. Sie könnte als Standardwerk für zukünftige Arbeiten dienen, die konkreter einzelne, hier oft nur angerissene Fragen, Schwerpunkte, Gegenstände ausloten werden. 